\title{
Long-range fractal correlations in literary corpora
}

\author{
Marcelo A. Montemurrd and Pedro A. Puryt \\ Facultad de Matemática, Astronomía y Física \\ Universidad Nacional de Córdoba \\ Ciudad Universitaria, 5000 Córdoba, Argentina
}

(October 18, 2018)

\begin{abstract}
In this paper we analyse the fractal structure of long human-language records by mapping large samples of texts onto time series. The particular mapping set up in this work is inspired on linguistic basis in the sense that is retains the word as the fundamental unit of communication. The results confirm that beyond the short-range correlations resulting from syntactic rules acting at sentence level, long-range structures emerge in large written language samples that give rise to long-range correlations in the use of words.
\end{abstract}

\section{INTRODUCTION}

The human language faculty reveals as a phenomenon of remarkable complexity, intimately interwoven with all our superior mental functions [1]. Its role in the modelling and categorisation of factual experience by the mind cannot be downplayed. Moreover, it has been suggested that the outburst of symbolic and abstract imagery pervading the archeological records signalling the transition between the middle and upper Paleolithic periods, might be interpreted as the hallmark of an associated, and rather sudden, transition in the linguistic performance of modern humans [2,3]. The evolutionary advantage of complex syntactic communication stems from the capacity that it confers to language of coding complex information using moderate symbolic resources [t. In the traditional areas of linguistics much success has been attained in dissecting language structure from sentence level down the morphological rules of word formation. However, effective linguistic communication is a complex cognitive process 5 where sequences of sentences cohere in the assemblage of larger meaningful structures. At this point the study of language becomes susceptible of being complemented by using techniques that have been successfully applied on other complex systems in nature that also allow a variety of interdisciplinary approaches.

In particular, one question that still remains open to discussion is the ultimate origin of long-range correlations in complex information systems like the genetic code or human language. The main goal of this work is to examine the existence of long-range correlations in the use of words in written language drawing on methods from statistical time series analysis. To that end, we first describe the particular mapping scheme used to translate the given sequence of words present in a text sample into a time series. Then, we devote a section to explain the rescaled range analysis and how it can be used to infer the presence of long-range correlations in time series. Finally, the results of our systematic analysis are presented followed by concluding remarks.

\section{THE MAPPING OF TEXTS ONTO TIME SERIES}

The statistical analysis of long symbolic structures had an accelerated development right after the first complete DNA sequences started to become available, around a decade ago [6]. Basically, the original techniques tried to create a random walk out of the particular symbolic sequence by mapping each distinct symbol onto a step in an independent direction in space. Thus, in a genetic sequence, made up of the concatenation of four independent nucleotides, represented by four letters, each instance of these symbols was translated onto an elementary jump along independent directions in a four dimensional space. Although different alternatives for the particular details of the mappings were put forward, all were devised to keep the original sequence's structure at symbol level translated into the time series. Then, by applying a set of standard statistical techniques, such as power spectrum analysis, detrended fluctuation analysis or rescaled range analysis among others, it was possible to unravel the existence of long-range correlations in biosequences [6, 7].

Written samples of natural languages, are also complex information carriers to be read sequentially. Thus, similar techniques as those employed in genetic sequence analysis were adapted with appropriate modifications 8 10]. The different procedures shared one common point, namely, that the mappings were invariably performed at letter level. However, it has never been discussed in the literature so far whether the mapping at letter level is indeed adequate in the case of human language. It is crucial to note that the specific coding of words in some particular spelling or phonetic system is alien to the linguistic structure of communication, as is clear by noting that a given text can be readily coded in sign languages, for instance. Therefore, a better mapping, founded on linguistics basis, should recognise the symbols at the minimum level intrinsic to the communication process. In what follows we propose the simplest 
step in that direction, which consists in taking word tokens as the fundamental units of communication. By doing this we can assert that the mapped sequences do not carry any structure below word level.

In order to make clear the details of the actual translation of a given text onto a time series, a brief detour around Zipf's analysis is required [11]. It basically consists in counting the number of occurrences of each distinct word in a given text sample, and then producing a list of all these words ordered by decreasing frequency. Each word in this list can be identified by an index equal to its rank, $r$, in the list, that is, the most frequent word has index $r=1$, the second in the list is given $r=2$, and so on. Words that occur the same number of times are ordered arbitrarily in their corresponding rank interval.

By means of Zipf's analysis each word in the original text can be replaced by its corresponding index $r$. Then, at position $t$ starting from the beginning of the text, we have the corresponding index $r(t)$. According to this mapping, the whole text becomes a time series of ranks, namely $\{r(t)\}_{t=1}^{T}$, where $T$ stands as the total length of the text. This particular numerical assignment may seem arbitrary at first, and in fact a different choice would certainly work as well. However, the selection of the rank as an indexing key to the words may be rendered more natural if we think of it as the assignment that minimises the effort in lexical access in the rankordered list of words in writing the whole text. When a word is required at position $t$ in the text, then it must be picked from position $r(t)$ in the list of words ordered by decreasing frequency.

In order to compare different time series generated by this mapping, it is useful to rescale the series to zero mean and unit variance. Thence, we define the standard deviation of the rank sample values as follows $\hat{\sigma}=\sqrt{\left\langle r(t)^{2}\right\rangle-\langle r(t)\rangle^{2}}$, where the symbol $\langle\ldots\rangle$ denotes an arithmetic average over the whole series of ranks. Then, the quantity $\xi(t)$ is defined as

$$
\xi(t)=\frac{r(t)-\langle r(t)\rangle}{\hat{\sigma}} .
$$

In this way, the sequence of normalised increments $\xi(t)$ can now be regarded as the sequence of elementary jumps in a random walk each taken at a discrete time $t$.

\section{RESCALED RANGE ANALYSIS}

Hydrology is the oldest discipline in which the presence of noncyclic very long-term dependence has been reported. Particularly, the rescaled range analysis was introduced by Harold E. Hurst [12] when he was studying the Nile in order to describe the long-term dependence of water levels in the river and reservoirs. Later, this statistical technique was further developed and applied by Mandelbrot and Wallis 13 15.
Let $\xi(t), 1 \leq t \leq T$, be the normalised sequence of increments of a process in discrete time. In our case, $\xi(t)$ is the normalised ordered sequence of ranks from a text corpus of $T$ words, as described in the previuos section. From this sequence, the record

$$
X(t)=\sum_{u=1}^{t} \xi(u)
$$

is constructed. Viewing the $\xi(t)$ as spatial increments in a one-dimensional discrete random walk, $X(t)$ is the position of the walker from the starting point at time $t$. For any given integer span $s>1$ and any initial time $t$, a detrended subrecord $D(u, t, s)$, for $0 \leq u \leq s$, can be defined as

$$
D(u, t, s)=X(t+u)-X(t)-\frac{u}{s}(X(t+s)-X(t)) .
$$

In this quantity, the mean $\sum_{w=1}^{s} \xi(t+w) / s$ was substracted to remove the trend in the subrecord. The cumulated range $R(t, s)$ of the subrecord is defined by

$$
R(t, s)=\max _{0 \leq u \leq s} D(u, t, s)-\min _{0 \leq u \leq s} D(u, t, s)
$$

and the variance $S^{2}(t, s)$ of the subrecord is defined by

$$
S^{2}(t, s)=\frac{1}{s} \sum_{w=1}^{s} \xi^{2}(t+w)-\left(\frac{1}{s} \sum_{w=1}^{s} \xi(t+w)\right)^{2}
$$

For many time series of natural phenomena the average of the sample values of $R(t, s) / S(t, s)$, carried over all admissible starting points $t$ within the sample, follows the Hurst's law: $\mathcal{E}[R(t, s) / S(t, s)] \sim s^{H}$ with $H>1 / 2$. Hurst's observation is remarkable considering the fact that in the absence of long-run statistical dependence one should find $H=1 / 2$, for processes with finite variance. For example, for a stationary Gaussian process $\xi(t)$ with $\langle\xi(t)\rangle=0$ and $\left\langle\xi^{2}(t)\right\rangle=1$, Feller [16] has analytically proved that

$$
\lim _{s \rightarrow \infty} s^{-1 / 2} \mathcal{E}[R(t, s) / S(t, s)]=\sqrt{\frac{\pi}{2}} .
$$

Additionally, Mandelbrot and Wallis [15] showed that the $s^{1 / 2}$ law also applies to processes of independent increments having a variety of distributions: truncated Gaussian, hyperbolic, and (highly skewed) log-normal. Moreover, they also showed that when the increments of the process are statistical dependent but the dependence is limited to the short run, the $s^{1 / 2}$ law holds asymptotically. The effect of strong cyclic components was also studied. When a white Gaussian noise (of zero mean and unit variance) is superimposed with a purely periodic process of amplitude $A$, it can be seen that 


$$
\lim _{s \rightarrow \infty} s^{-1 / 2} \mathcal{E}[R(t, s) / S(t, s)]=\sqrt{\frac{\pi}{2}}\left(1+\frac{A}{2}\right)^{-1 / 2} .
$$

The value of the limit as well as the speed with which this limit is attained is dependent on $A$. When $A$ increases, $\mathcal{E}[R(t, s) / S(t, s)]$ takes an even longer time to reach the $s^{1 / 2}$ law. Moreover, the transition to the asymptote is non monotonic, but typically exhibits a series of oscillations.

The above comments support the key idea that $s^{1 / 2}$ law holds for every process for which long-term dependence is unquestionably absent and does not hold for processes exhibiting noncyclic long-term statistical dependence. Thus, a Hurst exponent of $H=1 / 2$ corresponds to the vanishing of correlations between past and future spatial increments in the record. For $H>1 / 2$ one has persistent behaviour, which means a positive increment in the past will on the average lead to a positive increment in the future. Conversely a decreasing trend in the past implies on the average a sustained decrease in the future. Correspondingly, the case $H<1 / 2$ denotes antipersistent behaviour.

In all the above discussion the focus was on the scaling properties of the ratio $R(t, s) / S(t, s)$. It is important to note that the introduction of the denominator $S(t, s)$ becomes ever more relevant for processes that deviate from the Gaussian and/or have long-term dependence [15]. Futhermore, the ratio $R(t, s) / S(t, s)$ has a better sampling stability in the sense that the relative deviation, defined by $\sqrt{\operatorname{Var}[R(t, s) / S(t, s)]} / \mathcal{E}[R(t, s) / S(t, s)]$, is smaller than any alternative expression used to study long-term dependence.

\section{ESTIMATION OF $H$ IN LITERARY CORPORA}

In this section we shall explain the implementation of the rescaled range analysis, as well as present the results obtained after performing the experiments on texts coded as a normalised sequences of ranks.

As it was stated in the previous section, the method is based on the estimation of sample averages of the ratio $R(t, s) / S(t, s)$, for proper choice of different values of the integer span $s$. We shall follow the nomenclature and prescriptions introduced by Mandelbrot and Wallis 14] in the construction of the $\mathrm{R} / \mathrm{S}$ diagrams from the experimental data. We first compute the exponent $m$ such that $2^{m} \leq T<2^{m+1}$, and then we select the value of the span $s$ from the decreasing sequence of integers: $\left\{T / 2^{p}, p=0,1, \ldots, m-2\right\}$. For each $s$, we choose the starting points: $t=q s+1, q=0,1, \ldots, 2^{p}-1$ in order to construct $2^{p}$ nonoverlapping detrended subrecords $D(u, t, s)$ from the Eq. (3.2). Thus, for a given value of the index $p$, we have specified a value of $\log s$ which is marked on the axis of abscissas. In this way, we can reckon $2^{p}$ values of $\log [R(t, s) / S(t, s)]$ corresponding to the different starting points $t$, which are plotted as ordinates (marked by + signs). For each $s$, the logarithm of the sample average of the quantities $R(t, s) / S(t, s)$ over the different starting points is also marked in the $\mathrm{R} / \mathrm{S}$ diagram. Then, the value of $H$ is estimated by linear regression of $\log \mathcal{E}[R(t, s) / S(t, s)]$ vs. $\log s$. The slope is calculated using the linear least-square method and the error is evaluated taking the uncertainty in the ordinates, associated with each value of the abscissa $(\log s)$, equal to one quarter of the amplitude between the corresponding extreme points.

There are two pitfalls related to the $\mathrm{R} / \mathrm{S}$ diagrams. First, for small values of $\log s$ (short subrecords) there is large scattering in the values of $\log [R(t, s) / S(t, s)]$. Second, for large values of $\log s$ we have very few nonoverlapping subrecords, thus narrowing the R/S diagram. This tightening involves a deceiving evaluation of uncertainty in the ordinate. Hence, when fitting a straight trendline, small and large values of $\log s$ should be neglected. Given a value of $p$ we have $2^{p}$ subsamples each of which have $T / 2^{p} \geq 2^{m-p}$ ranks. Therefore, we will retain the values of the span $s$ given by $4 \leq p \leq m-4$ as our criterion for fitting. In this manner, we will only consider average over 16 or more samples and each subrecord will have at least 16 ranks.

\section{A. Results from literary corpora}

In Fig. 1 we show the R/S diagram corresponding to the coded sequence consisting in 885534 ranks from 36 plays by William Shakespeare. The total number of different ranks, associated with different words in the corpus, is in this case equal to 23150 . The mean and standard deviation $(\hat{\sigma})$ of the original sequence and the third (M3) and fourth (M4) moments of the normalised sequence are shown in an inset. For simplicity's sake, we only mark in the diagrams the minimum and maximum points for a given $\log s$, and the corresponding sample average is plotted as a small circle. The trendline is drawn as a solid line along the points effectively used in the fitting. The measured value of $H$ and its error are displayed in Table [1, and confirm the presence of long-range correlation in the series. In the same figure, we can also see as small squares the diagram for the sample average from the sequence after deleting all ranks outside the interval $(100,2000)$. Strikingly, the corresponding value of $H$ is statistically indistinguishable from that of the original sequence. This fact tells us that the core of long-range correlation is neither supported by the most frequent words nor the least used.

There are two additional experiments which can provide information in tracking down the source of the correlated behaviour. The first one is a simple random shuffling of all the ranks in the sequence which has the effect of recasting Shakespeare's plays into a nonsensical 
realisation, keeping the same original words without discernible order at any level. As is clear, after this experiment the analysis must yield a Hurst exponent indicating the uncorrelated character of the sequence. This is corroborated also in Fig. 11 where the sample average is plotted as small triangles. Yet more interesting is the analysis of a shuffling of Shakespeare's plays that preserves sentence structure, and therefore English grammar. That is, by defining a sentence as the sequence of words between two periods, we can reorder them in a random fashion and thus produce a grammatically correct, though hardly meaningful, version of the corpus. The sample averages are plotted in this case as small diamonds. The resulting value of $H$ shows that grammar is not sufficient to induce long-range correlations as we can see again from Table If. Let us note that for maintaining the readability of the graph we only included the extreme $\mathrm{R} / \mathrm{S}$ points of the original sequence.

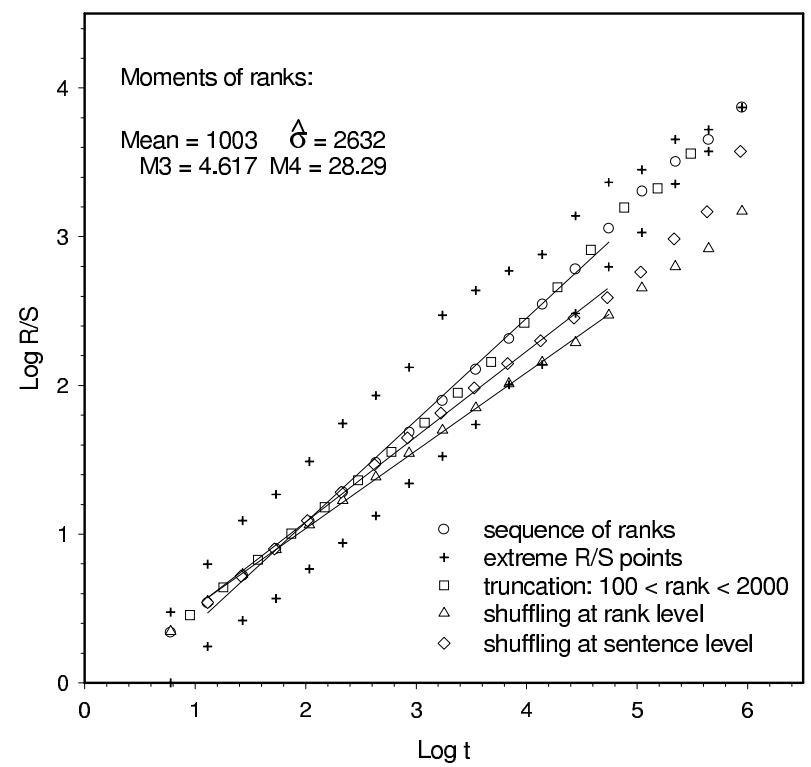

FIG. 1. R/S diagram corresponding to the Shakespeare corpus. The linear fits are showed only for the original sequence and the shuffling experiments.
At this point it may be illustrative to compare a plot of the record $X(t)$, the position of the walker as a function of time, both for the Shakespeare's plays and for a stochastic sequence generated with $H=0.7$ by the successive random addition method [17]. As can be seen in Figs. 2 and 3 both records show strong persistence that manifests itself in the long spans of average monotonous behaviour in the records.

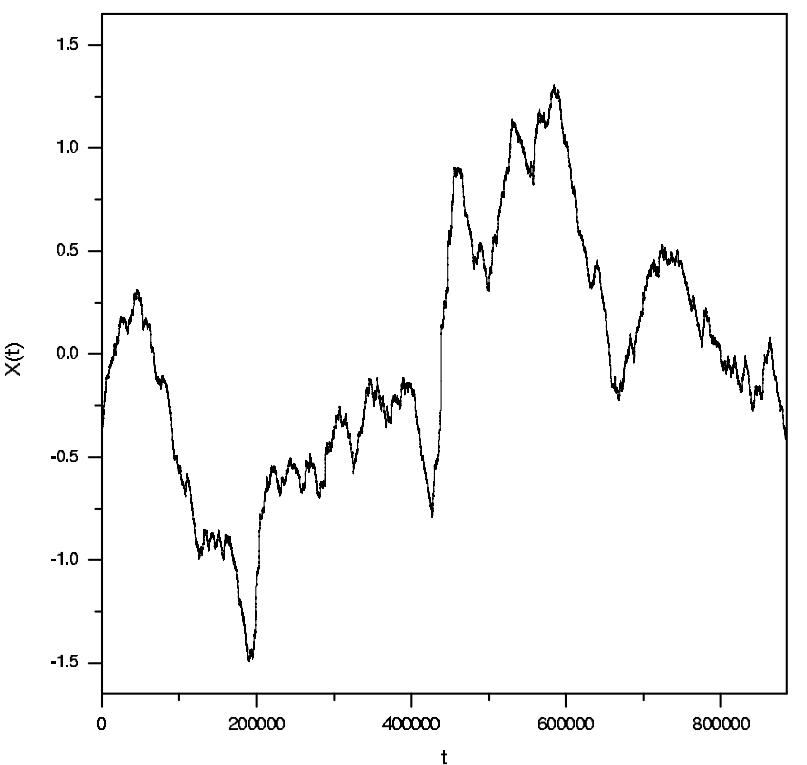

FIG. 2. Record of the coded sequence from the Shakespeare corpus.

\begin{tabular}{lccc}
\hline \hline source & original sequence & truncation $^{\mathrm{f}}$ & sentences shuffled $^{\text {ranks shuffled }}$ \\
Shakespeare $^{\mathrm{a}}$ & $0.687 \pm 0.040$ & $0.658 \pm 0.036$ & $0.574 \pm 0.035$ \\
Dickens $^{\mathrm{b}}$ & $0.738 \pm 0.033$ & $0.660 \pm 0.034$ & $0.573 \pm 0.025$ \\
Darwin $^{\mathrm{c}}$ & $0.745 \pm 0.045$ & $0.678 \pm 0.043$ & $0.576 \pm 0.033$ \\
Simon's model $^{\mathrm{d}}$ & $0.550 \pm 0.040$ & $0.519 \pm 0.032^{\mathrm{g}}$ & \\
Markovian text $^{\mathrm{e}}$ & $0.533 \pm 0.028$ & & \\
\hline \hline
\end{tabular}

TABLE I. Values of $H$ from the estimation by linear regression of $\log \mathcal{E}[R / S]$ vs. $\log s$.

a36 plays: 885534 words

b56 books: 5616403 words

${ }^{\mathrm{c}} 11$ books: 1508483 words

${ }^{\mathrm{d}} 5 \times 10^{6}$ words generated after a transient and deleting the ranks $\leq 5$

${ }^{\mathrm{e}} 1.2 \times 10^{6}$ words generated from table of frequencies corresponding to the Shakespeare corpus with memory of 7 letters

${ }^{\mathrm{f}}$ Unless other specification all ranks outside the interval $100<$ rank $<2000$ were deleted from the coded sequence

${ }^{\mathrm{g}}$ In this case the ranks outside the interval $5<\operatorname{rank}<10000$ were deleted 


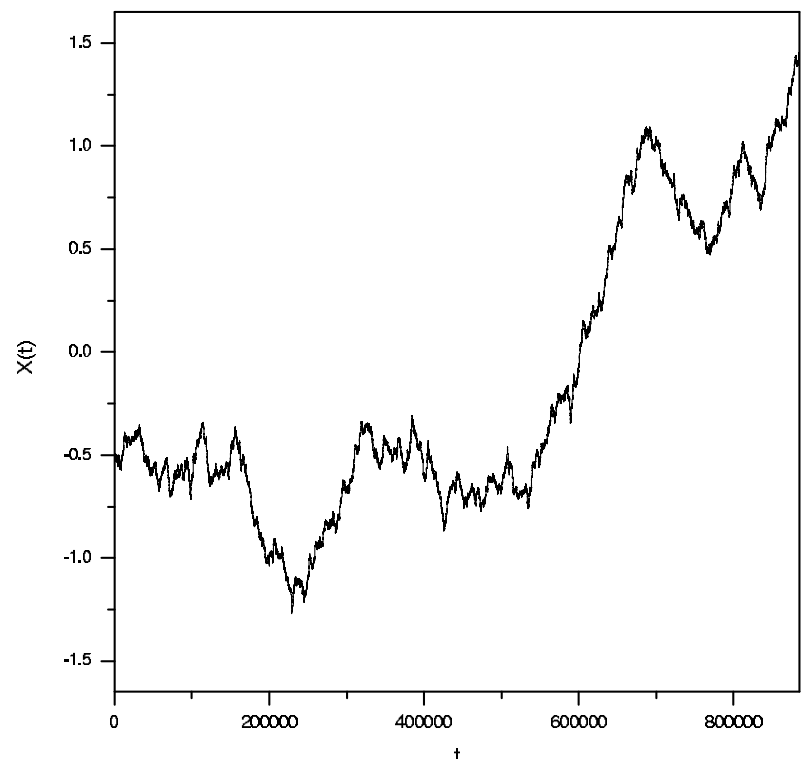

FIG. 3. Record of a fractional brownian motion generated with $H=0.7$

In Figs. 1 and 5 we reproduce an identical analysis from two text corpora gathering a collection of works by Dickens and Darwin respectively, whose results are summarised in Table Ii. The Dickens sequence was obtained from 56 books by the author, and has 5616403 ranks (words) in length (44700 different ranks), whereas the Darwin sequence was obtained from 11 books and has a length of 1508483 ranks (30120 words in the vocabulary). It is worth noticing that the values of $H$ from the original sequences are indistinguishable for these two texts, written in prose and with different styles, although they are slightly greater than the value obtained for the Shakespeare sequence. However, the three values of $H$ corresponding to the truncation $(100<\operatorname{rank}<2000)$ are statistically equivalent. This fact suggests that the long-range correlations associated to words in the interval considered are a robust phenomenon over different styles and authors. Finally, the shuffling experiments show the same behaviour for the three authors, and are consistent with uncorrelated sequences.

\begin{tabular}{lcc}
\hline \hline source & Shakespeare & Dickens \\
\hline corpus & $0.687 \pm 0.040$ & $0.738 \pm 0.033$ \\
portion & $0.675 \pm 0.044$ & $0.715 \pm 0.043$ \\
& $0.672 \pm 0.041$ & \\
\hline
\end{tabular}

TABLE II. Test of consistence: We estimated the $H$ value for portions of two corpora considered in Table If. The portions from the Shakespeare corpus correspond to the first and second half of the original source. From the Dickens corpus we have taken an embedded succession of 861038 words from an arbitrary origin in the original corpus.

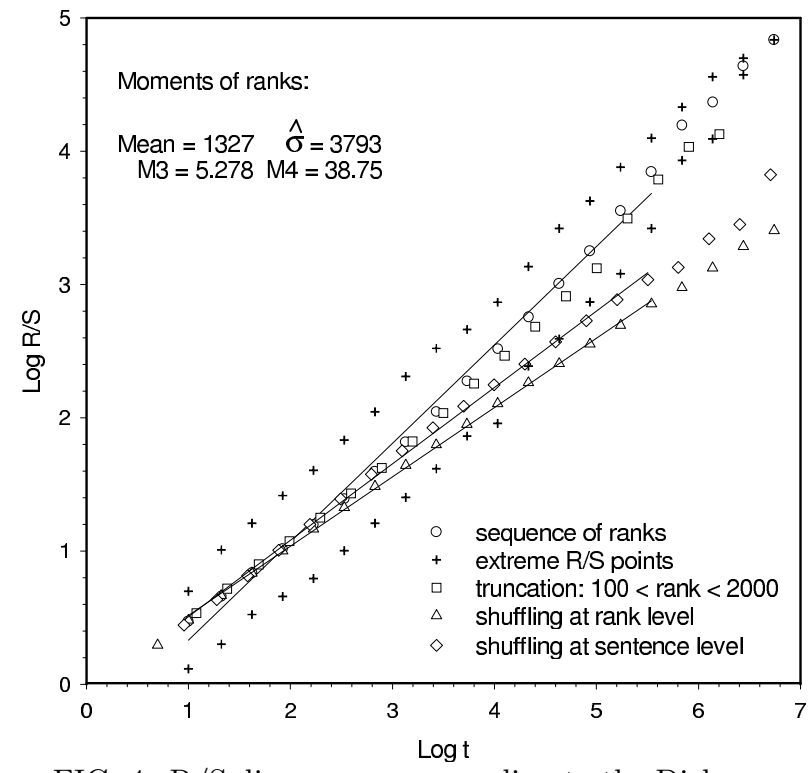

FIG. 4. R/S diagram corresponding to the Dickens corpus. The linear fits are showed only for the original sequence and the shuffling experiments.

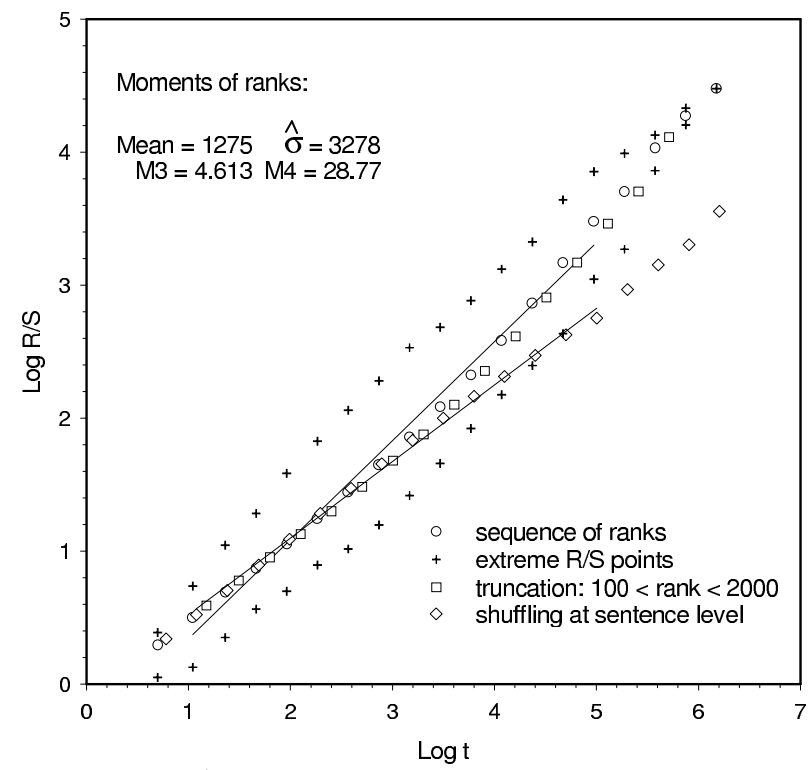

FIG. 5. R/S diagram corresponding to the Darwin corpus. The linear fits are showed only for the original sequence and the shuffling at sentence level.

\section{B. Tests of consistence}

In this subsection we present three tests of consistency of our analysis. First, we performed the same constructions as developed in the previous subsection over portions extracted from the original text corpora. Thus, we split the Shakespeare corpus into two parts of equal length and measured $H$ for each part. On the other hand, from the Dickens corpus we extracted a succession of 861038 words from an arbitrary origin and then 
measured the corresponding value of $H$. In Table $\amalg$ the values from both the original corpora and the parts are confronted. Values indistinguishable of $H$ result from the original corpus and its portions. In interpreting this result we should have in mind that Zipf's analysis of a portion of the text generates a quite different table of ranks from the one corresponding to the entire corpus. This serves as an indication that we are quantifying a robust phenomenon inherent to the fractal structure of texts.

In the insets of Figs. 1 and a we present the values of mean and standard deviation $(\hat{\sigma})$ corresponding to the set of ranks in the entire corpus of Shakespeare and Dickens respectively. For the Shakespeare corpus we can read that the mean of ranks is equal to 1003 , whereas $\hat{\sigma}=2632$. On the other hand, Fig. 4 shows for the Dickens corpus that the mean of ranks is equal to 1327 and $\hat{\sigma}=3793$. These quantities are calculated from sample values of a probability distribution $P(r)$, for which Zipf's law represents a crude approximation. However, by using more accurate analytical descriptions of the probability density $P(r)$, it is possible to evaluate the statistical moments for the whole distribution, that is, in the limit of infinite vocabulary. In particular, let us mention that the standard deviation calculated for both the Shakespeare and Dickens corpora, using the analytical expressions for $P(r)$ obtained in Ref. [18], result finite in the infinite vocabulary limit. The reason for this is that for large values of $r$, the probability density develops a fast exponential decay in the case of single-author corpora 18 .

In Table we also report the values of $H$ corresponding to corpora generated by means of stochastic processes. Particularly, we did the analysis explained above over a sequence of ranks generated by the Simon's model [19] and other corresponding to a Markovian text [20], with a memory of seven letters. This short-range memory is enough to string out groups of few words in grammatical order. However, as we can see from the values on the table, we do not obtain correlations from neither type of sequence, which is consistent with the expected behaviour for processes with short-range correlations. Consequently, our implementation of the rescaled range analysis allows to clearly distinguish between real and stochastic version of texts [21].

\section{The Dictionary}

So far, we still do not have enough evidence as to assert a precise source for the phenomenon being analysed in this paper. We have characterised with a robust quantifier the presence of long-range correlations in literary texts beyond sentence level, and in fact over ranges spanning more than one individual work. The shuffling experiments attest where the long-range correlation does not originate, but say nothing on where it does. In previous attempts to study this phenomenon, a variety of possi- ble origins for the long-range dependence have been put forward, though the conclusions in all cases have been inferred from the observed statistical behaviour of single literary works, and from the mapping of texts at letter level. In the present work we use a robust mapping with a strong footing on linguistics, and focus on the correlations that arise in large corpora comprising many individual books with no thematic linkage. Therefore, the existence of correlations overarching sets of entire works should be attributed to something else than either the relation between ideas expressed by the author as it was proposed in Ref. [8], or nonuniformities in the distribution of word's lengths and the associated densities of blank spaces as it was suggested in Ref. 10. In particular, the latter alternative is ruled out from the outset since our mapping does not carry information on word structure.

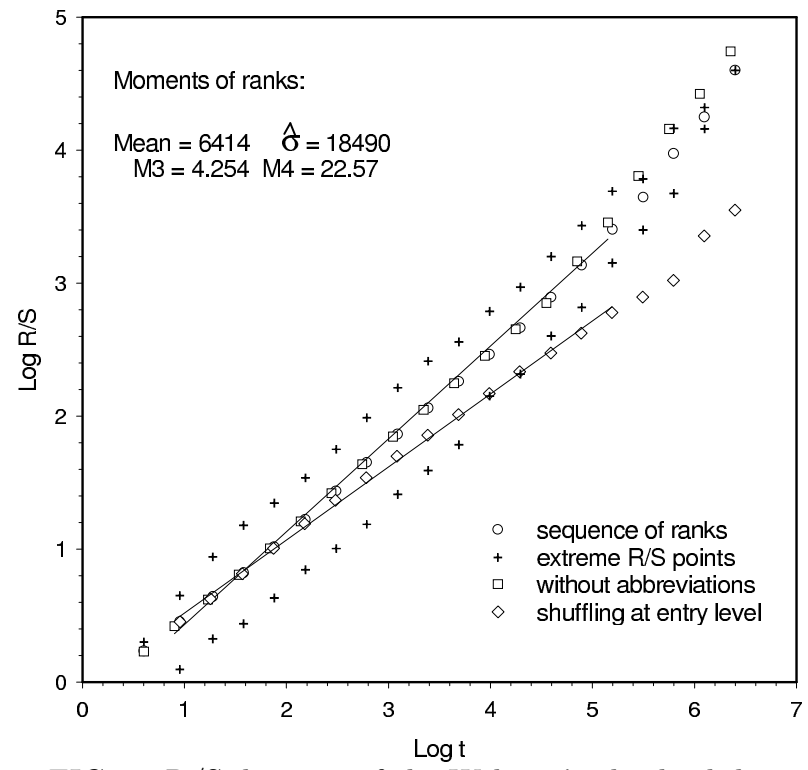

FIG. 6. R/S diagram of the Webster's abridged dictionary (1913 edition). The linear fits correspond to the sequences without the abbreviations.

In order to gain more insight into the origin of the attested long-range correlations, we decided to perform another experiment with a special type of text. A dictionary is a large collection of entries, one for each different word, arranged in alphabetical order. Clearly, in general, sequences of thematic affinity span at most a few entries, for small groups of words associated by common roots. Nevertheless, as is confirmed in Fig. 6 where we display the $\mathrm{R} / \mathrm{S}$ diagram generated from the Webster's abridged dictionary (1913 edition), the value of $H$ certifies the presence of long-range correlations. Table III compiles the exponent $H$ from the entire dictionary and from the text without the abbreviations (which indicate the grammatical category of entry words), obtaining in both cases totally consistent values for $H$. This result is rather striking and hints at the presence of a type of long-range organisation in the layout of lexical entries that may be con- 
nected with the phenomenon observed in literary works. This last assertion is further supported by performing a shuffle over the whole dictionary keeping integrity at entry level. That is, we do not disrupt the structure of entries made up of each word together with the associated definition, but we do mix them in a random manner. The resulting collection of definitions now lacks the alphabetical ordering; however, the explicit information contained in the dictionary is still intact. Notwithstanding, the long-range order is completely obliterated by the shuffle as accounted for by the fall in the $H$ value down to that of an uncorrelated sequence. This experiment corroborates that beyond the local structures derived from semantic affinity of small groups of related words, the ordered lexicon also possesses an overall macrostructure that emerges, as a hidden layer, out of the alphabetical order of entries.

A close examination at the structure of the dictionary reveals the source of the long-range order. Let us take for example the following sequence of related entries from the Webster's abridged dictionary (1913 edition): advice, advisability, advisable, advisableness, advisably, advise, advised, advising, advisedly, advisedness, advisement, adviser, advisership, advisor, and advisory. These entries form a small cohesive block of information, and all their definitions share many words. In turn, these shared terms point to, possibly, remote locations in the dictionary where, again, words with common roots form small clusters. This process carries on to many levels of depth, thus building a complex network of relations at word level, which may be closely related to the presence of long-range correlation in the succession of words in the dictionary. The entry-level shuffling destroys that emerging order, and thereby the correlations.

\begin{tabular}{|c|c|}
\hline Webster's Dictionary & $H$ \\
\hline original sequence & $0.690 \pm 0.031$ \\
\hline sequence without abbr. & $0.699 \pm 0.036$ \\
\hline entries shuffled ${ }^{a}$ & $0.548 \pm 0.025$ \\
\hline
\end{tabular}

TABLE III. Values of $H$ for the Webster's abridged dictionary (edition 1913) after coding as sequence of ranks.

${ }^{\mathrm{a}}$ from the text without abbreviations

\section{CONCLUSIONS}

In this work we have addressed the important issue of the emergence of long-range correlations in human written communication. To that end we proposed a simple mapping of texts onto random walks that keeps the word as the basic unit of communication. Therefore, the time series generated by this mapping retain the structure relevant to the linguistic phenomenon being analysed.

We addressed in some detail the rescaled range analysis, which has been successfully applied to the analysis of a vast variety of time series exhibiting long-term correlations. By applying this analysis to the coded texts we found conclusive evidence for long-range correlation in the use of words over spans as long as the whole corpora under study. It is worth noticing that the corpora used in this work are made up of the concatenation of independent literary works by individual authors, and, therefore the long-range effects must emerge as a phenomenon independent of the particular bounds of single literary works. This observation led us to analyse the case of a dictionary as a special kind of text, which provided insight into possible sources for the long-range order. The alphabetical order of entries in a dictionary corresponds only to the first visible layer of structural organisation. Yet, by performing the shuffling at entry level we realised that the whole of the long-range order is dependent upon a more complex structural layer related to the network of associations among word clusters.

As it has been convincingly shown in Ref. 22] sets of literary works also possess higher level structures associated with systematic patterns in word usage, which might also give rise to a complex network topology of relations among groups of words. The detailed mechanisms whereby the onset of these structures takes place in language requires further interdisciplinary research. In the light of the evidence supplied in this work, it is plausible that the ultimate source of these correlations is deeply related to the structural patterns of word distribution in written communication. In this view, groups of words associated by affine semantic hierarchies form a complex arrangement of cohesive clusters even over spans of entire corpora, thereby giving rise to long-range order in human written records.

\section{ACKNOWLEDGMENTS}

The authors are grateful to D. H. Zanette for his critical reading of the manuscript and to F. A. Tamarit for useful suggestions. This work has been partially supported by "Secretaría de Ciencia y Tecnología de la Universidad Nacional de Córdoba" (fellowship and grant 194/00). The digital texts analysed in this paper were obtained from Project Gutenberg Etext [23] 
* $\quad$ Electronic mail: mmontemu@famaf.unc.edu.ar

$\dagger \quad$ Electronic mail: pury@famaf.unc.edu.ar

[1] S. Pinker, The Language Instinct (Harper Collins, New York, 2000).

[2] P. Mellars, in The Origin and Diversification of Language, eds. N. G. Jabloski and L. C. Aiello (California Academy of Sciences, San Francisco, 1998)

[3] I. Tattersall and J. H. Matternes, Sci. Am. 282, 38 (January 2000).

[4] M. A. Nowak, J. B. Plotkin, and V. A. A. Jansen, Nature 404, 495 (2000).

[5] A. Aknajian, R. A. Demers, A. K. Farmer, and R. M. Harnish, Linguistics. An Introduction to Language and Communication (MIT Press, Cambridge, 1992).

[6] C.-K. Peng, S. V. Buldyrev, A. L. Goldberger, S. Havlin, F. Sciortino, M. Simons, and H. E. Stanley, Nature 356, 168 (1992); Richard F. Voss, Phys. Rev. Lett. 68, 3805 (1992); S. V. Buldyrev and A. L. Goldberger, S. Havlin, C.-K. Peng, M. Simons, F. Sciortino, and H. E. Stanley, ibid. 71, 1776 (1993); R. F. Voss, ibid. 71, 1777 (1993).

[7] Richard F. Voss, Fractals 2, 1 (1994).

[8] Alain Schenkel, Jun Zhang, and Yi-Cheng Zhang, Fractals 1, 47 (1993).

[9] M. Amit, Y. Shmerler, E. Eisenberg, M. Abraham, and N. Shnerb, Fractals 2, 7 (1994).

[10] Werner Ebeling and Thorsten Pöschel, Europhys. Lett. 26, 241 (1994); Werner Ebeling and Alexander Neiman, Physica A 215, 233 (1995).

[11] G. K. Zipf, Human Behavior and the Principle of Least Effort (Addison-Wesley, Reading, 1949).

[12] H. E. Hurst, Trans. Amer. Soc. Civil Eng. 116, 770 (1951).

[13] B. B. Mandelbrot and J. R. Wallis, Water Resources Research 5, 242 (1969).

[14] B. B. Mandelbrot and J. R. Wallis, Water Resources Research 5, 321 (1969).

[15] B. B. Mandelbrot and J. R. Wallis, Water Resources Research 5, 967 (1969).

[16] W. Feller, Ann. Math. Stat. 22, 427 (1951).

[17] R. F. Voss, Random Fractal Forgeries in Fundamentals Algorithms in Computer Graphics, eds. R. Pynn and A. Skjeltorp (Plenum Press, New York, 1985), pp. 1-11.

[18] Marcelo A. Montemurro, Physica A 300, 557 (2001)

[19] Herbert A. Simon, Biometrika 42, 425 (1955).

[20] Brian Hayes, Sci. Am. 249, 16 (November 1983).

[21] A. Cohen and R. N. Mantegna and S. Havlin, Fractals 5, 95 (1996).

[22] M. A. Montemurro and D. H. Zanette, Advances in Complex Systems, in press.

[23] http://promo.net/pg 